\title{
The importance of along-margin terrane transport in northern Gondwana: insights from detrital zircon parentage in Neoproterozoic rocks from Iberia and Brittany
}

\author{
J. Fernández-Suárez ${ }^{\mathrm{a} ;},{ }^{*}$ G. Gutiérrez Alonso ${ }^{\mathrm{b}}$, T.E. Jeffries ${ }^{\mathrm{c}}$ \\ ${ }^{a}$ Departamento de Petrología y Geoquímica Universidad Complutense, 28040 Madrid, Spain \\ bepartamento de Geología Universidad de Salamanca,33708Salamanca, Spain \\ c Department of Mineralogy, The Natural History Museum, London SW7 5BD, UK
}

\begin{abstract}
Detrital zircons from late Neoproterozoic rocks of the peri-Gondwanan Cadomian belt of SW Iberia and north Armorican Domain of Brittany record Neoproterozoic (ca. 860-550 Ma), Palaeoproterozoic (ca. 2300-1800) and Archaean (ca 3300-2600 Ma) U-Pb ages. The absence of Mesoproterozoic zircons suggests that these terranes evolved in a peri-W African realm. This is in contrast to other western European terranes that preserve Mesoproterozoic zircons and are likely to have evolved in a peri-Amazonian realm. Such a contrast in detrital zircon populations, coupled with the presence of Mesoproterozoic zircons in the Ordovician Armorican quartzite, deposited in a peri- African platform, is interpreted to record along-margin terrane transport. The change in provenance suggests that subduction was replaced by transform faults that juxtaposed Amazonia-derived terranes against W Africa-derived terranes to form the Avalonia and Armorica microcontinents. Subsequent extension along the margin resulted in the birth of the Rheic Ocean and the outboard drift of Avalonia.
\end{abstract}

?2002Elsevier Science B.V. Allrights reserved.

\section{Introduction}

Orogenic processes in active continental mar- gins involve major tectonic and thermal events leading to the creation, destruction and recycling of continental and oceanic crust, and the accretion, amalgamation and dispersal of crustal frag ments on different scales of space and time.

\footnotetext{
* Corresponding author. Tel.: +34-91-394-5013; Fax: $+34-91-544-2535$.

E-mail address: jfsuarez@geo.ucm.es (J. Fernández-Suárez).
}

Plate reorganisation at continental margins is often accompanied by substantial erosion and concomitant deposition of voluminous clastic sediments containing crucial information on the nature of the crustal elements in the provenance area.

The nature of plate processes and their importance in ancient orogens is commonly obscured by their involvement in subsequent tectonic events that destroy previous plate configurations. Overcoming this difficulty is particularly challenging in orogenic belts that have undergone more than one 
episode of dispersal and accretion. This is the case for the Neoproterozoic Avalonian-Cadomian orogenic belt [1] that was dismembered in Cambro-Ordovician times with the opening of the Rheic Ocean. Subsequently, it became involved in the late Palaeozoic VariscanAlleghanian orogeny, and finally, was dispersed again to its present-day arrangement in western Europe and eastern North America after the opening of the Atlantic Ocean.

Fundamental to our understanding and interpretation of these issues is the extent to which transform continental margins infiuence major crustal growth episodes and orogenic events [2]. Present-day tectonic settings such as the continental margin of western North America show the importance of along-margin terrane transport [2]. This has far-reaching implications for palaeo-geographic and plate reconstructions of ancient orogenic belts. In the case of early Palaeozoic and Precambrian orogenic belts, evidence for these processes is hard to find in the geological record. Even palaeomagnetic studies may fail to provide evidence for such processes as they may operate atscales of hundreds to afew thousands of $\mathrm{km}$.

Nevertheless, valuable information concerning major tectonic events and former plate configurations, as well as constraints on the nature and age of basement source rocks, can be obtained by comparing U-Pb detrital zircon ages and/or Sm-Nd isotope signatures in coeval sedimentary rocks from separate terranes [3-10]. Here we use this approach to address first-order Neoproterozoic-early Palaeozoic tectonic events along the margin of north Gondwana taking key Cadomian-Avalonian terranes of the western European Variscan Beltasacasestudy.

\subsection{Geology and palaeogeographic background}

The late Palaeozoic Variscan collision belt of western Europe contains a mixture of crustal blocks with Neoproterozoic basement that origi- nally formed part of an assemblage of terranes along the periGondwanan Cadomian-Avalonian arc [1,10]. It is generally agreed that these terranes formed as a result of Neoproterozoic subduction and arc construction along the northern Gond- wanan margin and that they were subsequently dispersed by the opening of the early Palaeozoic Rheic Ocean as subduction was replaced by transform/extensional activity [11,12]. Subsequent Palaeozoic plate tectonic motions that culminated in Appalachian-Variscan collisional orogenesis fur- ther redistributed these terranes toward their present arrangement in western Europe and Atlantic North Armorica. Reconstructing the tectonic evolution of these terranes and their location along the CadomianAvalonian orogen is fundamental to our understanding of major Neoproterozoic and early Palaeozoic tectonic events.

Palaeomagnetic studies, in addition to strati- graphic and palaeontological data, have provided a wealth of information on Neoproterozoic-early Palaeozoic continental reconstructions [13-15]. Yet, tighter constraints are needed in order to obtain a more detailed picture of the geography and tectonic setting of the northern Gondwanan margin and adjacent regions. Tracing the ancestry and evolution of the peri-Gondwanan terranes is further hindered by the absence of exposed preNeoproterozoic basement throughout most of the Cadomian-Avalonian Belt.

This study is focused on the Cadomian-Avalo- nian terranes presently exposed within the western European Variscan Belt (Fig. 1). Of particular interest are terranes such as Iberia and the Ar- morican Massif which feature major structural contacts between diferent tectonostratigraphic domains (Fig. 1). The origin and possible significance of these tectonic boundaries in the pre-Variscan evolution of these terranes are still poorly understood.

Restoration of the late Palaeozoic Iberian-Ar- morican Arc (Fig. 1) highlights the correlation of the Ossa Morena (SW Iberia) and the North American (Brittany) domains based on shared Neo- proterozoic-Lower Palaeozoic stratigraphy and abundance of igneous rocks formed during the time period ca 650-540 Ma [16]. Both domains record widespread Cadomian deformation and both are juxtaposed against the Central Iberian and South Armorican domains, respectively (Fig. 1). This juxtaposition occurs along major strike-slip faults with movement histories that 


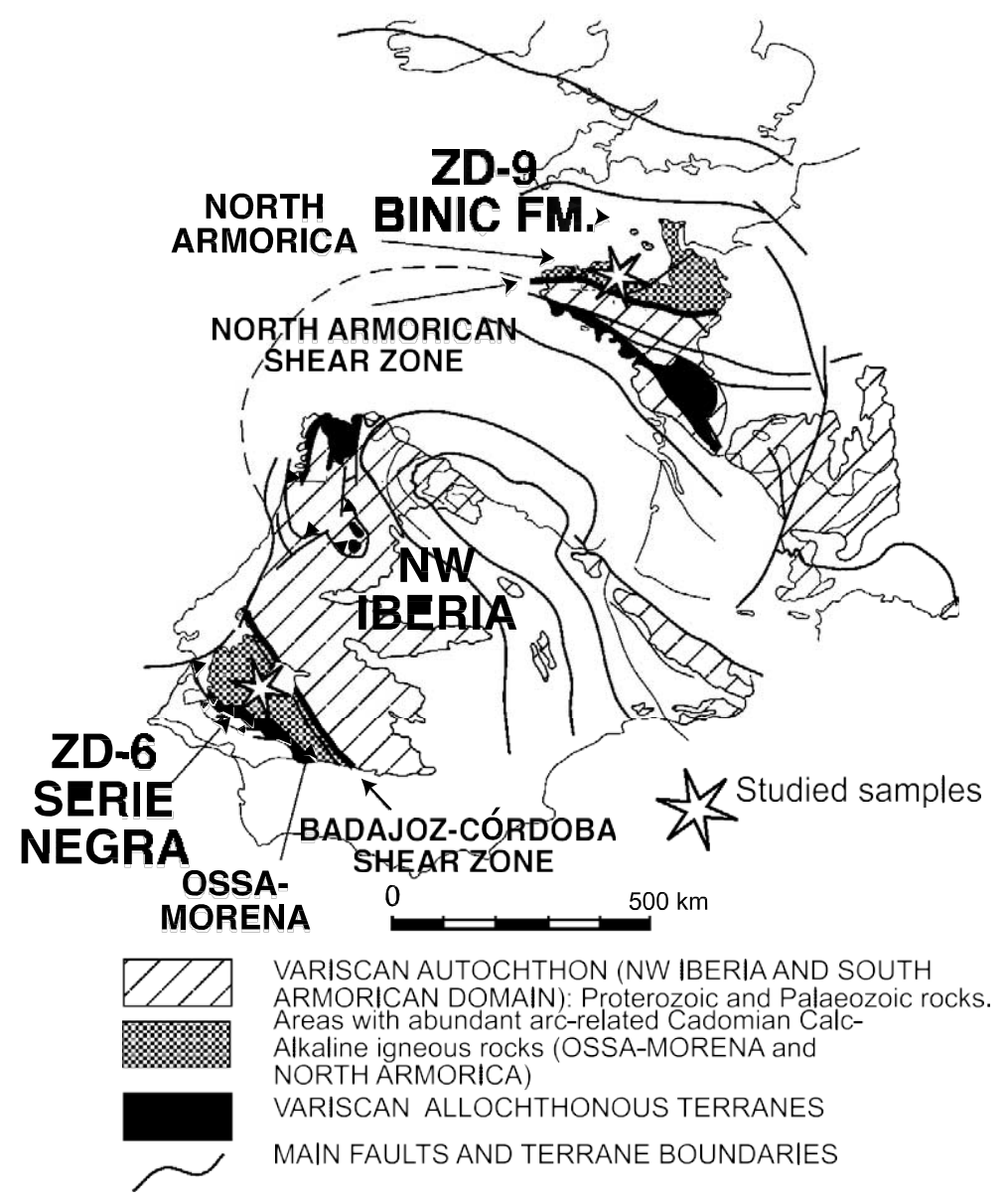

Fig. 1. Simplified geological map of Iberia and Cadomia showing location of samples ZD-6 (Ossa Morena, SW Spain) and ZD-9 (Brittany, NW France).

may predate the earliest recognised Variscan de- formation [16]. To test this correlation, two sam- ples were collected from low-grade greywackes in both domains (Fig. 1) in order to compare the age populations of their detrital zircons with those in the NW Iberian Domain $[6,17]$. Sample ZD-6 was collected in the Ossa Morena Zone (Province of Badajoz, SW Spain), in the upper part of the Serie Negra, in the core of the Olivenza-Monesterio antiform (Fig. 1). Sample ZD-9 was collected from Brioverian sediments of the Binic Forma- tion, north of the town of Binic (Brittany, NW France). Both formations are considered to be of late Neoproterozoic depositional age [18].

Sample ZD-6 is a coarse-grained heterometric lithic greywacke containing clasts of quartz, igne- ous rocks, micas and plagioclase in a matrix of finegrained phyllosilicates with a coarse cleavage (pressure-solution seams). Sample ZD-9 is a medium-grained arkosic greywacke containing quartz, plagioclase and scarce igneous rock frag- ments in a matrix of recrystallised phyllosilicates that display a poorly developedcleavage.

\section{U-Pb analytical methods}

Mineral separation was performed at UCM (Madrid). The samples were crushed using a jaw crusher and pulverised with a disc mill. Heavy fraction enrichment on a Wilfley table, magnetic separation using a Frantz isodynamic separator 
and density separation using di-iodomethane $\left(\mathrm{CH}_{2} \mathrm{I}_{2}\right)$ were employed to separate the zircons. The zircons were hand-picked in alcohol under a binocular microscope and grains chosen to repre- sent all types found in the samples with regard to size, length-to-breadth ratio, roundness, colour, and other salient morphological features. Zircons selected for LA-ICP-MS analyses were set in syn- thetic resin (SpeciFix-201) mounts, polished to approximately half their thickness, and cleaned in a warm $\mathrm{HNO}_{3}$ ultrasonic bath. Prior to analysis, cathodoluminiscence (CL) and backscattered electron (BSE) imaging was performed to ensure that analyses did not straddle diferent domains as indicated by the structure revealed by BSE and CL imaging.

$\mathrm{U}-\mathrm{Pb}$ dating of individual zircon grains was performed using $213 \mathrm{~nm}$ laser ablation ICP-MS at The Natural History Museum (London). The $213 \mathrm{~nm}$ laser system had not been used previously to determine U-Pb isotopic ratios in zircons, and prior to its application to the unknown samples, the technique was validated and refined using zir- con standards and igneous zircons previously dated by IDTIMS. The main advantages of the $213 \mathrm{~nm}$ laser over the more widely used $266 \mathrm{~nm}$ laser are a slower, more stable ablation rate and the production of consistently small, readily trans- ported ablated particles. This results in increased signal stability and precision in the analyses. More detailed information on the features of ablation using a $213 \mathrm{~nm}$ laser is given in $[19,20]$. In view of the relatively large ablation pit size (typically 50-60 Wm), only zircons considered to be homogeneous on the basis of their CL/BSE images, or large cores, were analysed.

The instrumentation used consisted of a fre- quency quintupled Nd:YAG laser ablation sys- tem, $V=213$ nm (New Wave Research, USA) coupled to a quadrupole based ICP-MS (Plasma- Quad 3, Thermo Elemental, UK) with an en- hanced sensitivity (Soption) interface. In this study, analyses were performed using argon as carrier gas. It is worth noting that a work in progress by the authors shows that the use of helium carrier gas greatly improves sample trans- port e/ciency, which reduces elemental fraction- ation and results in more concordant analyses. It hasno efect on theprecision of measured ${ }^{207} \mathrm{~Pb} /{ }^{206} \mathrm{~Pb}$ ratios.

To reduce the extent of inter-element laser-in- duced fractionation, the zircons were ablated at the lowest power density required to couple to the sample (pulse energy $=0.15 \mathrm{~mJ}$ per pulse). During ablation, the sample was moved relative to the beam along raster or line patterns, using a computer-controlledmotorisedstage.

Each analytical 'run' consisted of 20 analyses in the following order: four analyses of standard reference zircon 91500 [21], 12 analyses of the sample zircons and four analyses of standard 91500. After each analytical session, the raw data were downloaded to a PC for of-line pro- cessing. Raw count rates were integrated by aver- aging consecutive groups of 15 sweeps into single readings. The data were then processed using LAMTRACE, a data-reducing programme for la- ser ablation ICP-MS written by Simon E. Jack- son, Macquarie University, Sydney, Australia. Net background-corrected count rates for each isotope were used for calculation of sample ages. Age and error calculation are as described in $[6,8]$ with two exceptions: (i) we use the standard reference zircon 91500 instead of the 02123 in-house standard, and (ii) we use measured ${ }^{207} \mathrm{~Pb} /{ }^{235} \mathrm{U}$ ratios in our calculations. The data reported have internal precision (2c) based on counting statistics of each individual analysis.

For each determination, time-resolved signals were carefully studied to select stable, non-fractionated intervals, ensuring that inclusions, zona- tion or core-rim features were always excluded from age calculations.

\section{Results}

A total of 120 analyses were performed on sin- gle grains (one analysis per grain) from both sam- ples. Of these, 37 were rejected based on the cri- teria reported below. The results for selected analyses are given in Table 1 and presented as concordia diagrams and histograms in Figs. 2 and 3, respectively.

Although the analytical methodology (see above) ensures ablation of homogeneous zircon 
Table 1

Laserablation ICP-MSU-Pbanalyses(concordantanalysesinbold type)

\begin{tabular}{|c|c|c|c|c|c|c|c|}
\hline \multirow[t]{2}{*}{ Samp./Anal.\# } & \multicolumn{3}{|c|}{ Isotopic ratios (2c errors) } & \multicolumn{3}{|l|}{ Age (Ma) } & \multirow{2}{*}{$\begin{array}{l}\text { Best age estimate } \\
(\mathrm{Ma})\end{array}$} \\
\hline & ${ }^{206} \mathrm{~Pb} /{ }^{238} \mathrm{U}$ & ${ }^{207} \mathrm{~Pb} /{ }^{235} \mathrm{U}$ & ${ }^{207} \mathrm{~Pb} /{ }^{206} \mathrm{~Pb}$ & ${ }^{206} \mathrm{~Pb} /{ }^{238} \mathrm{U}$ & ${ }^{207} \mathrm{~Pb} /{ }^{235} \mathrm{U}$ & ${ }^{207} \mathrm{~Pb} /{ }^{206} \mathrm{~Pb}$ & \\
\hline \multicolumn{8}{|l|}{ ZD-6 } \\
\hline $\mathrm{Z1}$ & $0.0794 \times 0.0004$ & $0.6511 \times 0.0036$ & 0.0594 X 0.0002 & $493 \times 4$ & $509 \times 4$ & $582 \times 16$ & $582 X 16$ \\
\hline $\mathrm{Z2}$ & $0.0836 \times 0.0006$ & $0.6810 \times 0.0057$ & $0.0591 \times 0.0003$ & $518 \times 8$ & $527 \times 7$ & $568 \times 22$ & $568 \times 22$ \\
\hline Z3 & $0.0838 \times 0.0007$ & $0.6936 \times 0.0044$ & $0.0600 \times 0.0003$ & $519 \times 8$ & $535 \times 5$ & $602 \times 24$ & $602 \times 24$ \\
\hline $\mathrm{Z} 4$ & $0.0838 \times 0.0005$ & $0.7029 \times 0.0063$ & $0.0608 \times 0.0004$ & $519 \times 6$ & $541 \times 7$ & $632 \times 30$ & $632 \times 30$ \\
\hline Z5 & $0.0856 \times 0.0005$ & $0.7067 \times 0.0048$ & $0.0599 \times 0.0003$ & $529 \times 6$ & $543 \times 6$ & $598 \times 20$ & $598 \times 20$ \\
\hline Z6 & $0.0872 \times 0.0005$ & $0.7086 \times 0.0047$ & $0.0589 \times 0.0003$ & $540 \times 6$ & $544 \times 6$ & $564 \times 22$ & $564 X 22$ \\
\hline $\mathrm{Z7}$ & $0.0875 \times 0.0005$ & $0.7069 \times 0.0060$ & $0.0586 \times 0.0005$ & $541 \times 5$ & $543 \times 7$ & $550 \times 20$ & $541 \pm 3$ \\
\hline Z8 & $0.0884 \times 0.0007$ & $0.7521 \times 0.0064$ & 0.0617 X 0.0003 & $546 \times 8$ & $569 \times 7$ & $662 \times 20$ & $662 \times 20$ \\
\hline Z9 & $0.0889 \times 0.0008$ & $0.7536 \times 0.0068$ & $0.0615 \times 0.0002$ & $549 \times 9$ & $570 \times 8$ & $654 X 16$ & $654 X 16$ \\
\hline Z10 & $0.0898 \times 0.0007$ & $0.7569 \times 0.0066$ & $0.0611 \times 0.0004$ & $554 \times 8$ & $572 X 8$ & $642 \times 30$ & $642 \times 30$ \\
\hline Z11 & $0.0898 \times 0.0004$ & $0.7418 \times 0.0034$ & $0.0599 \times 0.0003$ & $554 \times 5$ & $563 \times 4$ & $600 \times 20$ & $600 \times 20$ \\
\hline Z12 & $0.0926 \times 0.0007$ & $0.7542 \times 0.0067$ & 0.0591 X 0.0004 & $571 \times 9$ & $571 X 8$ & $568 \times 28$ & $571 \pm 4$ \\
\hline Z13 & $0.0928 \times 0.0008$ & $0.7541 \times 0.0075$ & $0.0589 \times 0.0003$ & $572 \times 9$ & $571 \times 9$ & $562 \times 22$ & $571 \pm 4$ \\
\hline Z14 & $0.0959 \times 0.0010$ & $0.7888 \times 0.0077$ & $0.0596 \times 0.0006$ & $591 X 12$ & $591 \times 9$ & $588 \times 42$ & $591 \pm 5$ \\
\hline Z15 & $0.0971 \times 0.0007$ & $0.7995 \times 0.0065$ & 0.0597 X 0.0003 & $597 \times 8$ & $597 \times 7$ & $592 \times 20$ & $597 \pm 4$ \\
\hline Z16 & $0.0990 \times 0.0012$ & $0.8188 \times 0.0094$ & $0.0600 \times 0.0004$ & $609 X 14$ & $607 X 11$ & $600 \times 32$ & $607 \pm 5$ \\
\hline Z17 & $0.1026 \times 0.0006$ & $0.8935 \times 0.0072$ & $0.0632 \times 0.0003$ & $629 \times 7$ & $648 \times 8$ & $714 X 22$ & $714 X 22$ \\
\hline Z18 & $0.1114 \times 0.0013$ & $0.9966 \times 0.0175$ & $0.0649 \times 0.0010$ & $681 X 15$ & $702 X 18$ & $768 \times 64$ & $768 \times 64$ \\
\hline Z19 & $0.1185 \times 0.0007$ & $1.1105 \times 0.0077$ & $0.0680 \times 0.0002$ & $722 \times 8$ & $758 \times 7$ & $866 \times 12$ & $866 \times 12$ \\
\hline Z20 & $0.1233 \times 0.0007$ & $1.1522 \times 0.0075$ & $0.0678 \times 0.0003$ & $749 \times 8$ & $778 \times 7$ & $860 \times 18$ & $860 \times 18$ \\
\hline Z21 & $0.2772 \times 0.0033$ & $4.3681 \times 0.0590$ & 0.1143 X 0.0005 & 1577X34 & $1706 \times 22$ & $1868 X 14$ & 1868X14 \\
\hline Z22 & $0.2824 \times 0.0020$ & $4.6787 \times 0.0426$ & 0.1201 X 0.0005 & $1603 X 20$ & $1763 X 15$ & $1958 \times 16$ & $1958 \times 16$ \\
\hline Z23 & $0.2909 \times 0.0025$ & $4.6014 \times 0.0428$ & 0.1147 X 0.0004 & $1646 X 25$ & $1750 \times 15$ & $1874 X 12$ & $1874 X 12$ \\
\hline Z24 & $0.2912 \times 0.0019$ & $4.6276 \times 0.0305$ & 0.1152 X 0.0004 & 1647X19 & 1754X11 & $1882 X 12$ & $1882 X 12$ \\
\hline Z25 & $0.3005 \times 0.0028$ & $5.3919 \times 0.1202$ & 0.1301 X 0.0026 & $1694 X 28$ & 1884X38 & 2098X68 & 2098X68 \\
\hline Z26 & 0.3025 X 0.0018 & $5.0316 \times 0.0327$ & 0.1206 X 0.0007 & $1704 X 17$ & 1825X11 & $1964 X 22$ & $1964 X 22$ \\
\hline Z27 & $0.3068 \times 0.0018$ & $5.3052 \times 0.0355$ & 0.1254 X 0.0003 & 1725X18 & $1870 X 11$ & $2034 X 8$ & $2034 X 8$ \\
\hline Z28 & $0.3104 \times 0.0026$ & $4.9953 \times 0.0495$ & 0.1167 X 0.0007 & $1743 X 25$ & 1819X17 & $1906 \times 22$ & $1906 \times 22$ \\
\hline Z29 & 0.3159 X 0.0027 & $5.3759 \times 0.0430$ & 0.1234 X 0.0003 & $1770 X 27$ & 1881X14 & $2004 \times 10$ & $2004 X 10$ \\
\hline Z30 & $0.3180 \times 0.0022$ & $5.8373 \times 0.0946$ & 0.1331 X 0.0018 & 1780X21 & $1952 X 28$ & $2138 X 48$ & $2138 X 48$ \\
\hline Z31 & 0.3342 X 0.0021 & $5.2517 \times 0.0373$ & $0.1140 \times 0.0003$ & $1859 X 21$ & $1861 X 12$ & $1862 \times 10$ & $1862 \pm 6$ \\
\hline Z32 & $0.3485 \times 0.0070$ & 5.5957 X 0.1158 & $0.1165 \times 0.0003$ & 1927X67 & 1915X36 & $1902 \times 10$ & $1902 \pm 8$ \\
\hline Z33 & 0.3487 X 0.0040 & $5.9122 \times 0.0656$ & 0.1229 X 0.0005 & 1929X38 & 1963X19 & 1998X14 & $1998 X 14$ \\
\hline Z34 & $0.3642 \times 0.0034$ & $6.5804 \times 0.0691$ & $0.1310 \times 0.0006$ & $2002 X 32$ & $2057 X 19$ & $2110 \times 16$ & $2110 \times 16$ \\
\hline Z35 & $0.3829 \times 0.0021$ & $7.0189 \times 0.0316$ & $0.1329 \times 0.0007$ & $2090 X 20$ & $2114 X 8$ & $2136 \times 16$ & $2136 X 16$ \\
\hline Z36 & $0.4043 \times 0.0033$ & $8.4528 \times 0.0718$ & $0.1516 \times 0.0004$ & 2189X30 & 2281X15 & $2364 X 10$ & $2364 X 10$ \\
\hline Z37 & $0.4513 \times 0.0039$ & 11.0716 X 0.1163 & $0.1779 \times 0.0006$ & $2401 X 35$ & $2529 X 20$ & $2632 \times 12$ & $2632 \times 12$ \\
\hline Z38 & $0.4582 \times 0.0049$ & 11.5137 X 0.1267 & 0.1822 X 0.0005 & $2431 X 43$ & 2566X21 & $2672 X 8$ & $2672 X 8$ \\
\hline Z39 & $0.5168 \times 0.0041$ & $16.2267 \times 0.1314$ & 0.2277 X 0.0004 & $2685 X 35$ & $2890 \times 15$ & $3034 X 6$ & $3034 \times 6$ \\
\hline $\mathrm{Z} 40$ & $0.5369 \times 0.0029$ & $16.9163 \times 0.0998$ & $0.2285 \times 0.0005$ & 2771X24 & 2930X11 & $3040 \times 6$ & $3040 \times 6$ \\
\hline Z41 & $0.5726 \times 0.0043$ & 21.3067 X 0.1385 & $0.2698 \times 0.0013$ & 2919X35 & 3153X13 & $3304 X 16$ & $3304 X 16$ \\
\hline Z42 & $0.6207 \times 0.0036$ & 22.9077 X 0.1489 & $0.2676 \times 0.0014$ & $3113 X 29$ & $3223 \times 13$ & $3292 \times 16$ & $3292 \times 16$ \\
\hline \multicolumn{8}{|l|}{ ZD-9 } \\
\hline Z43 & $0.0904 \times 0.0007$ & $0.7320 \times 0.0053$ & 0.0587 X 0.0002 & $558 \times 8$ & $558 \times 6$ & $556 \times 12$ & $558 \pm 4$ \\
\hline Z44 & 0.0894 X 0.0005 & $0.7324 \times 0.0050$ & $0.0594 \times 0.0003$ & $552 \times 6$ & $558 \times 6$ & $580 \times 22$ & $580 \times 22$ \\
\hline Z45 & 0.0874 X 0.0007 & $0.7221 \times 0.0061$ & 0.0599 X 0.0004 & $540 \times 8$ & $552 \times 7$ & $598 \times 30$ & $598 \times 30$ \\
\hline Z46 & $0.0898 \times 0.0006$ & $0.7466 \times 0.0051$ & $0.0603 \times 0.0002$ & $555 \times 7$ & $566 \times 6$ & $612 \times 12$ & $612 X 12$ \\
\hline Z47 & $0.0886 \times 0.0005$ & $0.7389 \times 0.0044$ & $0.0605 \times 0.0002$ & $547 X 5$ & $562 \times 5$ & $620 \times 18$ & $620 \times 18$ \\
\hline Z48 & $0.0878 \times 0.0008$ & $0.7320 \times 0.0072$ & $0.0605 \times 0.0003$ & $543 \times 9$ & $558 \times 8$ & $620 X 18$ & $620 X 18$ \\
\hline Z49 & 0.0904 X 0.0008 & $0.7573 \times 0.0064$ & 0.0607 X 0.0002 & $558 \times 9$ & $572 \times 7$ & $630 \times 18$ & $630 \times 18$ \\
\hline Z50 & $0.0863 \times 0.0006$ & $0.7234 \times 0.0072$ & $0.0608 \times 0.0004$ & $533 \times 7$ & $553 \times 8$ & $632 \times 26$ & $632 \times 26$ \\
\hline
\end{tabular}


Table 1 (Continued).

\begin{tabular}{|c|c|c|c|c|c|c|c|}
\hline \multirow[t]{2}{*}{ Samp./Anal.\# } & \multicolumn{3}{|c|}{ Isotopic ratios (2c errors) } & \multicolumn{3}{|l|}{ Age (Ma) } & \multirow{2}{*}{$\begin{array}{l}\text { Best age estimate } \\
\text { (Ma) }\end{array}$} \\
\hline & ${ }^{206} \mathrm{~Pb} /{ }^{238} \mathrm{U}$ & ${ }^{207} \mathrm{~Pb} /{ }^{235} \mathrm{U}$ & ${ }^{207} \mathrm{~Pb} /{ }^{206} \mathrm{~Pb}$ & ${ }^{206} \mathrm{~Pb} /{ }^{238} \mathrm{U}$ & ${ }^{207} \mathrm{~Pb} /{ }^{235} \mathrm{U}$ & ${ }^{207} \mathrm{~Pb} /{ }^{206} \mathrm{~Pb}$ & \\
\hline $\mathrm{Z} 51$ & $0.0934 \times 0.0005$ & $0.7849 \times 0.0050$ & $0.0610 \times 0.0003$ & $576 \times 5$ & $588 \times 6$ & $636 \times 24$ & $636 \times 24$ \\
\hline Z52 & $0.0974 \times 0.0009$ & $0.8219 \times 0.0081$ & $0.0612 \times 0.0001$ & $599 X 11$ & $609 \times 9$ & $646 \times 10$ & $646 \times 10$ \\
\hline Z53 & $0.0867 \times 0.0008$ & $0.7315 \times 0.0068$ & $0.0612 \times 0.0003$ & $536 \times 9$ & $557 X 8$ & $646 \times 18$ & $646 \times 18$ \\
\hline $\mathrm{Z} 54$ & $0.0915 \times 0.0013$ & $0.7754 \times 0.0118$ & $0.0614 \times 0.0004$ & $565 X 15$ & $583 X 13$ & $654 \times 32$ & $654 \times 32$ \\
\hline Z55 & $0.0898 \times 0.0009$ & $0.7633 \times 0.0082$ & $0.0616 \times 0.0006$ & $554 X 11$ & $576 \times 10$ & $660 \times 40$ & $660 \times 40$ \\
\hline Z56 & $0.0917 \times 0.0009$ & $0.7816 \times 0.0098$ & $0.0618 \times 0.0003$ & $566 X 11$ & $586 X 11$ & $666 \times 18$ & $666 \times 18$ \\
\hline $\mathrm{Z} 57$ & $0.0895 \times 0.0006$ & $0.7627 \times 0.0044$ & $0.0618 \times 0.0002$ & $552 \times 7$ & $576 \times 5$ & $666 \times 16$ & $666 \times 16$ \\
\hline Z58 & $0.0924 \times 0.0008$ & $0.7885 \times 0.0078$ & $0.0619 \times 0.0004$ & $569 \times 9$ & $590 \times 9$ & $670 \times 24$ & $670 \times 24$ \\
\hline Z59 & 0.1183 X 0.0012 & $1.0132 \times 0.0105$ & $0.0621 \times 0.0003$ & 721X14 & 711X11 & $676 \times 22$ & $676 \times 22$ \\
\hline $\mathrm{Z} 60$ & $0.0980 \times 0.0008$ & $0.8409 \times 0.0071$ & $0.0622 \times 0.0003$ & $603 \times 9$ & $620 \times 8$ & $682 \times 22$ & $682 \times 22$ \\
\hline Z61 & $0.0897 \times 0.0007$ & $0.7712 \times 0.0079$ & $0.0624 \times 0.0006$ & $554 \times 9$ & $580 \times 9$ & $686 \times 44$ & $686 \times 44$ \\
\hline Z62 & $0.1012 \times 0.0008$ & $0.8825 \times 0.0072$ & $0.0632 \times 0.0004$ & $622 \times 10$ & $642 \times 8$ & $714 X 28$ & $714 X 28$ \\
\hline Z63 & 0.1031 X 0.0008 & $0.9042 \times 0.0093$ & $0.0636 \times 0.0004$ & $632 \times 9$ & $654 X 10$ & $728 \times 24$ & $728 \times 24$ \\
\hline Z64 & $0.1092 \times 0.0005$ & $0.9851 \times 0.0126$ & $0.0654 \times 0.0006$ & $668 \times 6$ & $696 \times 13$ & $786 \times 40$ & $786 \times 40$ \\
\hline Z65 & $0.1115 \times 0.0007$ & $1.0195 \times 0.0076$ & $0.0663 \times 0.0003$ & $681 X 8$ & $714 X 8$ & $814 X 22$ & $814 X 22$ \\
\hline Z66 & $0.1299 \times 0.0008$ & $1.1972 \times 0.0104$ & $0.0669 \times 0.0006$ & $787 \times 9$ & $799 X 10$ & $832 X 38$ & $832 X 38$ \\
\hline Z67 & 0.1397 X 0.0010 & $1.2926 \times 0.0103$ & $0.0671 \times 0.0005$ & $843 X 12$ & $842 \times 9$ & $840 \times 32$ & $843 \pm 5$ \\
\hline Z68 & $0.2842 \times 0.0027$ & $4.7056 \times 0.0391$ & $0.1200 \times 0.0004$ & 1613X28 & $1768 X 14$ & $1956 X 12$ & $1956 X 12$ \\
\hline Z69 & $0.3123 \times 0.0013$ & $5.2156 \times 0.0235$ & $0.1211 \times 0.0004$ & $1752 X 13$ & $1855 X 8$ & $1972 \times 10$ & $1972 \times 10$ \\
\hline $\mathrm{Z70}$ & 0.3391 X 0.0013 & $5.8282 \times 0.0303$ & $0.1246 \times 0.0003$ & 1882X12 & $1951 X 9$ & $2022 \times 10$ & $2022 \times 10$ \\
\hline Z71 & $0.2954 \times 0.0020$ & 5.1531 X 0.0392 & $0.1265 \times 0.0002$ & $1669 \times 20$ & $1845 X 13$ & $2048 \times 6$ & $2048 \times 6$ \\
\hline $\mathrm{Z} 72$ & 0.2949 X 0.0021 & $5.2888 \times 0.0439$ & $0.1300 \times 0.0005$ & $1666 X 21$ & 1867X14 & $2098 \times 14$ & $2098 X 14$ \\
\hline Z73 & $0.3464 \times 0.0055$ & $6.5536 \times 0.1167$ & $0.1372 \times 0.0024$ & 1917X53 & 2053X31 & $2190 X 62$ & $2190 \times 62$ \\
\hline Z74 & $0.3710 \times 0.0029$ & $7.1334 \times 0.0578$ & $0.1395 \times 0.0002$ & $2034 X 27$ & $2128 X 14$ & $2220 X 6$ & $2220 \times 6$ \\
\hline $\mathrm{Z75}$ & $0.4170 \times 0.0025$ & $8.3922 \times 0.0814$ & $0.1460 \times 0.0010$ & $2247 X 23$ & $2274 X 18$ & $2298 \times 24$ & $2298 \times 24$ \\
\hline Z76 & $0.3812 \times 0.0032$ & 7.7401 X 0.0418 & $0.1473 \times 0.0007$ & 2082X30 & $2201 X 10$ & 2314X18 & $2314 X 18$ \\
\hline $\mathrm{Z77}$ & $0.5333 \times 0.0077$ & $12.4708 \times 0.1796$ & $0.1696 \times 0.0007$ & 2755X64 & $2641 X 27$ & $2552 X 14$ & $2552 \times 14$ \\
\hline Z78 & 0.4551 X 0.0090 & $11.0839 \times 0.2250$ & 0.1766 X 0.0011 & $2418 \times 80$ & 2530X38 & $2620 \times 22$ & $2620 \times 22$ \\
\hline Z79 & $0.4536 \times 0.0020$ & 11.2347 X 0.0528 & $0.1796 \times 0.0005$ & $2411 X 18$ & $2543 \times 9$ & $2648 X 8$ & $2648 X 8$ \\
\hline Z80 & $0.5299 \times 0.0039$ & 13.4333 X 0.0994 & $0.1839 \times 0.0009$ & 2741X33 & 2711X14 & $2686 \times 16$ & 2686X16 \\
\hline Z81 & $0.5418 \times 0.0046$ & $14.5080 \times 0.1190$ & $0.1942 \times 0.0004$ & 2791X38 & $2784 X 16$ & $2776 X 6$ & $2779 \pm 4$ \\
\hline Z82 & $0.5936 \times 0.0045$ & $19.7721 \times 0.1186$ & $0.2416 \times 0.0012$ & $3004 X 36$ & $3080 X 12$ & $3130 \times 16$ & $3130 \times 16$ \\
\hline Z83 & $0.7534 \times 0.0052$ & $28.8080 \times 0.1815$ & $0.2773 \times 0.0006$ & $3620 X 38$ & $3447 X 12$ & $3346 \times 6$ & $3346 X 6$ \\
\hline
\end{tabular}

domains, analyses with S $20 \%$ discordance (calculated from $\left[{ }^{206} \mathrm{~Pb} /{ }^{238} \mathrm{U}\right] /\left[{ }^{207} \mathrm{~Pb} /{ }^{206} \mathrm{~Pb}\right]$ age) were rejected. The time-resolved analysis software re- ports signal intensity (counts per second) for every mass sweep performed by the mass spec- trometer. This protocol, allowing acquisition of signals as a function of time (a proxy for ablation depth profile), permits the recognition of isotopic heterogeneity and therefore the detection of inclusions, high common $\mathrm{Pb}$ associated to fractures or areas of radiation damage, and inherited $\mathrm{Pb}$ components (older cores). All analyses whose time-resolved signals showed any suspect features were excluded from the data set irrespective of their discordance. Using this approach, it can be as- sumed confidently that discordance in the selected analyses is not due to mixing of diferently aged zircon domains but by lead loss and perhaps mi- nor U-Pb laser-induced elemental fractionation, undetectable in the time-resolved signals. Such fractionation does not afect the measured ${ }^{207} \mathrm{~Pb} /{ }^{206} \mathrm{~Pb}$ ratios.

The ages reported in this paper are not common Pbcorrected as ${ }^{204} \mathrm{~Pb}$ measurements are ren- dered useless by the isobaric interference from $\mathrm{Hg}$, a contaminant present in the argon supply gas. ${ }^{204} \mathrm{Hg}$ interferes on ${ }^{204} \mathrm{~Pb}$ and the ${ }^{202} \mathrm{Hg}$ peak is commonly too small to allow a reliable overlap correction of acceptable precision [22].

Since the LA-(quadrupole)-ICP-MS technique 

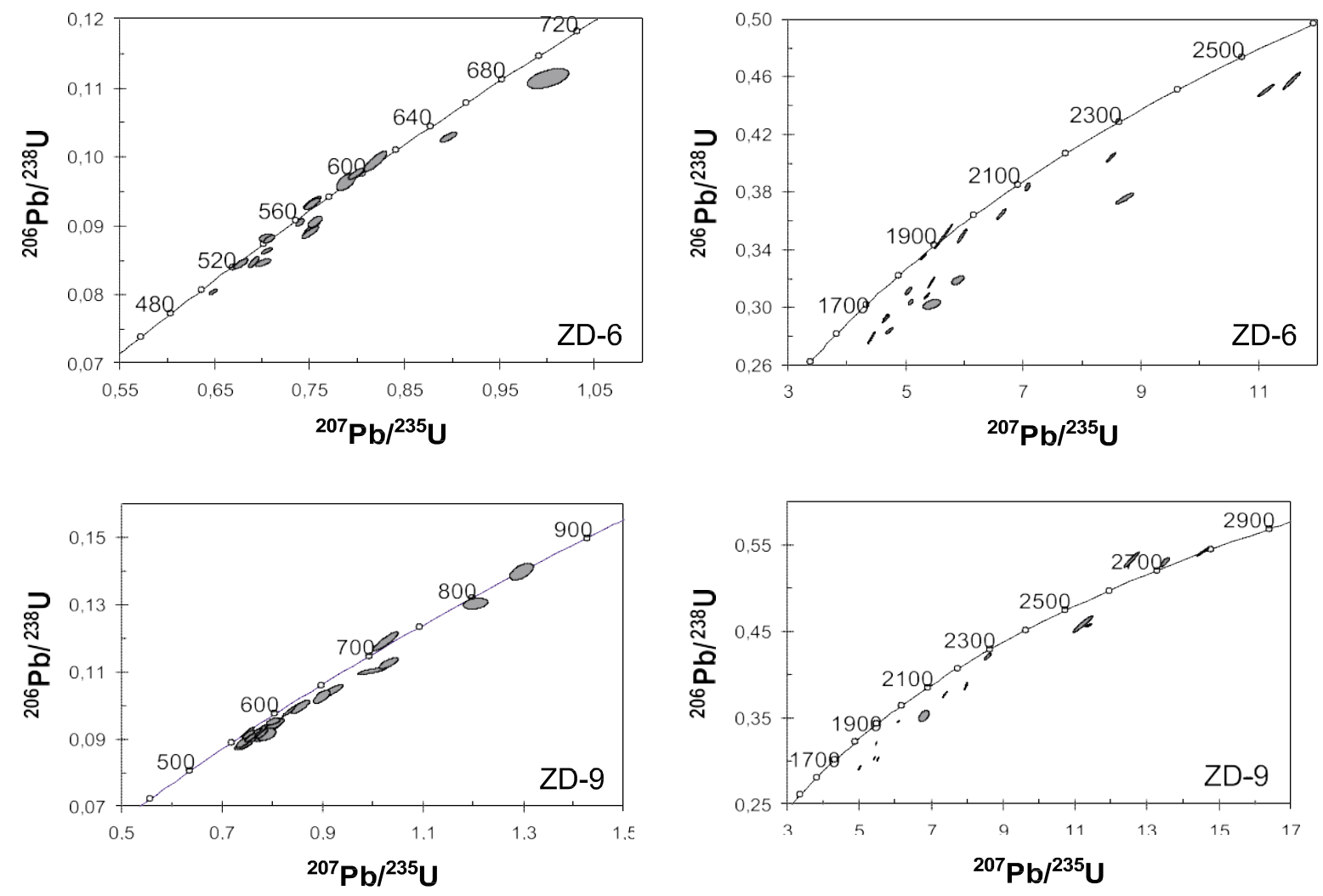

Fig. 2. Concordiaplots of U-Pbanalytical data from samples ZD-6andZD-9. Ellipses represent2c uncertainties.

using a natural zircon standard does not allow reliable determination of ${ }^{204} \mathrm{~Pb}$, an alternative op- tion would be to use the ${ }^{208} \mathrm{~Pb}$ correction [23]. However, this method can only be applied if the $\mathrm{ThO}^{\mathrm{p}} / \mathrm{UO}^{\mathrm{b}}$ ratio [23] can be simultaneously determined with acceptable precision or assuming concordance of ${ }^{206} \mathrm{~Pb} /{ }^{238} \mathrm{U}$ and ${ }^{208} \mathrm{~Pb} /{ }^{232} \mathrm{Th}$ ages. As the assumption cannot be justified a priori, it is felt that this correction method should not be applied to analyses such as those reported in this study (cf. [24]). The ${ }^{208} \mathrm{~Pb} /{ }^{232} \mathrm{Th}$ ratio (not reported in Table 1 ) is very sensitive to common $\mathrm{Pb}$ contamination, resulting in ${ }^{208} \mathrm{~Pb} /{ }^{232} \mathrm{Th}$ ages that are significantly older than the ${ }^{206} \mathrm{~Pb} /{ }^{238} \mathrm{U}$ ages. This feature ofers a qualitative criterion to detect zircons with high common $\mathrm{Pb}$ content in LAICP-MS analyses [9]. In this study we found this feature only in analyses where ${ }^{206} \mathrm{~Pb} /{ }^{238} \mathrm{U}-{ }^{207} \mathrm{~Pb} /{ }^{206} \mathrm{~Pb}$ discordance is S $20 \%$ and therefore would have been rejected irrespective of this fact.
Furthermore, it should be emphasised that most zircons do not contain significant amounts of common $\mathrm{Pb}$ and that for most Proterozoic zircons, common $\mathrm{Pb}$ corrections would be within the analytical uncertainty of the analyses reported here. For example, a SHRIMP study of zircons from a Neoproterozoic sedimentary formation of SW Iberia (stratigraphically equivalent to that in which sample ZD-6 was collected) [25] shows that the percentage of common ${ }^{206} \mathrm{~Pb}$ relative to total measured ${ }^{206} \mathrm{~Pb}$ is less than $0.5 \%$ in $80 \%$ of the analyses and $\mathrm{s} 1 \%$ in only $10 \%$ of the analyses reported. In [25] all analyses with significant amounts of common $\mathrm{Pb}$ are $\mathbf{S}$ 25\% discordant (usually with reverse discordance) and have high Th contents. Finally, it should be noted that the age clusters derived from common Pb-corrected U-Pb data in [25] coincide with those reported in this study for a formation of stratigraphic equivalence (see below). 


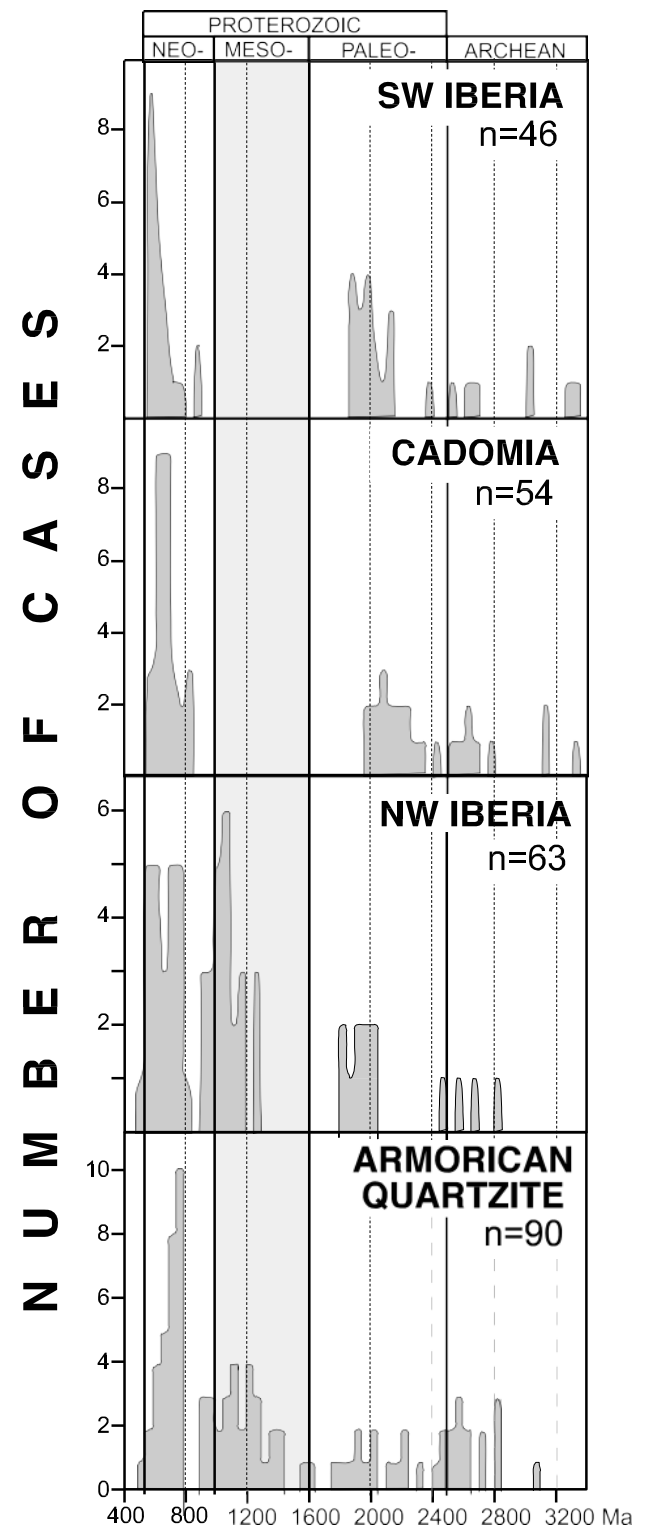

Fig. 3. Histograms of $\mathrm{U}-\mathrm{Pb}$ ages for analysed samples from Ossa Morena Zone, SW Iberia (ZD-6) and north Armorican Domain (ZD-9) compared to previously analysed samples from NW Iberia [6] and the Armorican quartzite [17].

Considering the above arguments, we are con-

fident that, in the carefully screened analyses re- ported here, only a very small percentage of the discordant ${ }^{207} \mathrm{~Pb} /{ }^{206} \mathrm{~Pb}$ ages might be slightly older than the 'correct age'. As the purpose of this study is to define age clusters in detrital zircon populations representing major crust formation events, the possible uncertainty associated with some of the discordant ages is insignificant to the interpretation of the data and has no influence on the main conclusions reached in this study. As a routine further precaution, those discordant analyses whose ${ }^{207} \mathrm{~Pb} /{ }^{206} \mathrm{~Pb}$ ages have no concordant counterparts are not used to derive geological conclusions, i.e. we only consider conclusions that would not be afected if solely ${ }^{207} \mathrm{~Pb} /{ }^{206} \mathrm{~Pb}$ or ${ }^{206} \mathrm{~Pb} /{ }^{238} \mathrm{U}$ ages were used. As is the case in this study, the reliability of detrital zircon LA-ICP- MS U-Pb data is strengthened by the reproduc- ibility of age patterns in samples that are considered equivalent on the basis of independent geological criteria [6].

In the data presented here, a few data points plot slightly above concordia (Fig. 2; i.e. the

${ }^{207} \mathrm{~Pb} /{ }^{206} \mathrm{~Pb}$ age is younger than the ${ }^{206} \mathrm{~Pb} /{ }^{238} \mathrm{U}$ and ${ }^{207} \mathrm{~Pb} /{ }^{235} \mathrm{U}$ ages). This phenomenon of reverse discordance is frequently observed in both SIMS and LAICP-MS U-Pb analyses and although not well understood, it is generally attributed to redis- tribution of radiogenic lead on a micron scale $[24,26]$ or associated with poor correction for $\mathrm{U}-\mathrm{Pb}$ fractionation (fractionation in the sample deviates significantly from that in the standard) [8]. In either case, the ${ }^{207} \mathrm{~Pb} /{ }^{206} \mathrm{~Pb}$ ratio gives the best age estimate as the efect is comparable to moving the point up or down a discordia line going through $0 \mathrm{Ma}$ (assuming that no other sources for discordance play a significant role).

The ages reported in the histograms of Fig. 3 and labelled 'best age estimate' in Table 1 are obtained as follows: For concordant analyses (ages whose corresponding isotope ratios have a 2c error ellipse that, to a greater or lesser extent, overlaps the concordia curve) we use concordia ages and errors as defined by Ludwig [27]. For discordant analyses we use the ${ }^{207} \mathrm{~Pb} /{ }^{206} \mathrm{~Pb}$ ages and 2c errors.

If we assume that discordant analyses can be attributed to $\mathrm{Pb}$ loss and since the efect of pos- sible episodic lead loss at intermediate ages can- not be evaluated, individual ${ }^{207} \mathrm{~Pb} /{ }^{206} \mathrm{~Pb}$ dates rep- resent minimum ages of growth for a given zircon. Again, as in these types of studies the clustering of grain ages is the crucial indicator of zircon- 
forming high-grade events and since the efect of $\mathrm{Pb}$ loss is to disperse zircon ages [4] (i.e. broad- ening the spectra), the presence of well-defined clusters must be considered geologically significant.

The U-Pb ages in sample ZD-6 (Ossa Morena Zone, SW Iberia) show a predominance of zircons in the range 540-860 Ma (Figs. 2A and 3). The youngest zircon has a ${ }^{206} \mathrm{~Pb} /{ }^{238} \mathrm{U}$ age of $541 \mathrm{X} 6 \mathrm{Ma}$ (concordia age of $541.4 \mathrm{X}$ $2.4 \mathrm{Ma}$ ) and a less precise ${ }^{207} \mathrm{~Pb} /{ }^{206} \mathrm{~Pb}$ age of $550 \mathrm{X} 20 \mathrm{Ma}$ (2c). Con- cordant zircon ages in this cluster spread between ca 541 and $608 \mathrm{Ma}$, whereas discordant ages ( 6 20\% discordance) spread from ca 560 to 866 Ma. Of these discordant analyses (Table 1 ) seven fall within the range of concordant analyses and eight have older ${ }^{207} \mathrm{~Pb} /{ }^{206} \mathrm{~Pb}$ ages from 632 to $866 \mathrm{Ma}$. Although no concordant zircons in this older range were dated in this study, Ordø nez Casado [25] reported common $\mathrm{Pb}$-corrected SHRIMP ages for zircons in a stratigraphically equivalent formation spanning541X19to847X26Ma.

The second zircon cluster is of Palaeoprotero- zoic age (ca 1860-2300 Ma; Table 1). The young- est zircon in this group is sub-concordant (2.2\% discordance) with a ${ }^{207} \mathrm{~Pb} /{ }^{206} \mathrm{~Pb}$ age of $1860 \mathrm{X} 10 \mathrm{Ma} .15$ zircons have ages ranging from ca 1860 to $2130 \mathrm{Ma}$ (Table 1, Figs. 2B and 3). One zircon yielded an older discordant ${ }^{207} \mathrm{~Pb} /{ }^{206} \mathrm{~Pb}$ age of 2364 X 10 Ma. Five zircons yielded ${ }^{207} \mathrm{~Pb} /{ }^{206} \mathrm{~Pb}$ Archaean ages in the range ca 2600-3300 Ma (Fig. 3) with discordance $612 \%$.

Zircon U-Pb ages in sample ZD-9 (Brittany) show a pattern similar to that of ZD-6 (Table 1, Figs. 2C,D and 3). The youngest age cluster is of Neoproterozoic age, ca 550-840 Ma. The young- est zircon in this group is concordant (30.4\% dis- cordance) with a ${ }^{206} \mathrm{~Pb} / 238 \mathrm{U}$ age of 558 X $8 \mathrm{Ma}$, within analytical error of the ${ }^{207} \mathrm{~Pb} /{ }^{206} \mathrm{~Pb}$ age of the youngest zircon in sample ZD-6. The oldest zircon in this cluster has a concordia age of $843 \times 5$ with $85 \%$ probability of concordance, very similar to the ${ }^{207} \mathrm{~Pb} /{ }^{206} \mathrm{~Pb}$ age of the oldest zircon of this group in sample ZD-6 (866X12 Ma).

The second most abundant group consists of Palaeoproterozoic zircons with ages ranging from ca 1950 to 2300 Ma (Table 1, Figs. 2D and 3). As in SW Iberia, a few zircons yielded Archaean ages (2550-3350 Ma). Each of these age groups is consistent with U-Pb IDTIMS sin gle-grain data from detrital zircons in the stratigraphically equivalent Jersey Shale Formation [28].

\section{Discussion}

\subsection{Significance of $U$-Pb ages}

The ages of the youngest detrital zircons in both formations are equivalent within analytical uncertainty, suggesting a coeval maximum late Neoproterozoic depositional age. These data are in agreement with other studies [18] and support their stratigraphic equivalence. This is an important constraint when interpreting detrital zircon ages, particularly at active continental margins like the Cadomian-Avalonian orogen, where palaeogeographic settings and source area exposures can vary significantly in short time intervals.

The similar $\mathrm{U}-\mathrm{Pb}$ age populations of the detrital zircons in late Neoproterozoic greywackes from SW Iberia and the North Armorican Domain both indicate provenance from a crustal source formed shortly before deposition and older cratonic sources. Those Neoproterozoic ages in the range ca 540-560 to 620-650 Ma match the span of the main episodes of magmatic activity related to subduction and arc construction along the northern Gondwanan margin (compilation in [29]). Zircons with Neoproterozoic ages older than ca $650 \mathrm{Ma}$ could have been generated either during the early arc phase $[6,11]$ or they could represent detritus from rocks generated in Neoproterozoic interior orogens related to the assembly of west Gondwana[30,31].

Palaeoproterozoic zircon ages match the spread of loosely constrained orogenic events recorded in the west African craton [32] and Amazonia [33,34]. The presence of these zircons in the studied rocks is in agreement with the existence of a Palaeoproterozoic (ca 1.8-2.1 Ga) basement in western Europe (Icartian basement) [35,36]. Archaean zircons dated in this study match the age spread of orogenic events recorded in cratonic 
areas of west Africa [37-39] and Amazonia [34,40]. Therefore, the U-Pb age clusters found in the studied rocks indicate a clear Gondwanan signature for these late Neoproterozoic sedimentary formations.

\subsection{Geodynamic implications}

The Cadomian-Avalonian terranes of western Europe and Atlantic North America were devel- oped upon, and recycled material from, a cratonic basement whose age and composition varied along the northern Gondwanan margin [1]. With the exception of the ca 2 Ga Icartian gneisses in Brittany and the Channel Islands [35,36], this basement is not exposed and insights into its age and composition must be gained indirectly through U-Pb and Sm-Nd isotopic studies of Neoproterozoic and early Palaeozoic rocks. Such studies have identified two main basement signatures; one with affinities to west Africa and the other with affinities to Amazonia. Characteristic of the latter is the presence of Mesoproterozoic zircons (1600-1000 Ma), coupled generally with higher initial $O_{\mathrm{Nd}}$ values (e.g. [1]).

The most significant feature of the detrital zircon ages from SW Iberia and Brittany is therefore the absence of Mesoproterozoic zircons. This contrasts with Neoproterozoic and early Palaeozoic sedimentary rocks in NW Iberia (Fig. 3) and other areas of the Central European Variscides (West Sudetes and Erzgebirge [41] and Moravo-Silesian Zone [42]), and with those reported from West Avalonia [1,5,29].

The lack of Mesoproterozoic (Grenvillian, Sun- sasRondonian) detrital zircons suggests that the SW Iberian and north Armorican fragments of the CadomianAvalonian orogen like most of the Saxothuringian and Moldanubian zones of central Europe which show similar detrital age characteristics [43,44] occupied a realm that had no access to exposures of basement rocks contain- ing Mesoproterozoic zircons. Of the likely cratonic provenances, only the west African craton lacks significant zircon-forming Mesoproterozoic events, suggesting that SW Iberia, the north Armorican Domain and parts of the Bohemian Massif prob- ably lay adjacent to this craton at that time (Fig.
4A). NW Iberia, NE Bohemia and Moravo-Silesia, on the other hand, require a Mesoproterozoic basement source, suggesting closer proximity to Amazonia or Oaxaquia [45] (Fig. 4A).

The contrast in sedimentary provenance be- tween west Africa and Amazonia is also re£ected in Sm-Nd isotope signatures. Rocks with west African anities show much lower initial $O_{\mathrm{Nd}}$ val- ues than those with Amazonian anity. For ex- ample, the Neoproterozoic greywackes from NW Iberia record initial $O_{\mathrm{Nd}}$ values between 0 and 35 [46], whereas greywackes of equivalent age in SW Iberia record initial $O_{\mathrm{Nd}}$ values between 39 and 312 [47].

All these terranes formed part of the active northern margin of Gondwana (Fig. 4A) that featured both a magmatic arc and a variety of back- arc basins (e.g. [11]). A back-arc setting has been proposed for both NW Iberia [6] and NE Bohemia [41]. These regions may have been closer to the Gondwanan margin than the West Avalonian terranes, which are dominated by magmatic arc rocks and likely represent the main magmatic ed- ifice on the Amazonian segment of the margin (Fig. 4A).

Subduction along the margin proceeded until either ridge-trench collision [11] or a change in subduction regime [6] caused progressive transition to a transform regime (Fig. 4B-D). The arrest of subduction is considered to have been diachronous along the margin (e.g. [11] and references therein). This is consistent with the contrasting evolution of SW Iberia and Brittany with respect to NW Iberia and the predominance of younger Neoproterozoic detrital zircons in rocks from Brittany and SW Iberia in relation to those from NW Iberia (Fig. $3)$.

In the western European Variscan Belt, terranes with difering (Amazonian versus west African) basement signatures were juxtaposed prior to the deposition of the Middle Ordovician Armorican quartzite [48], which oversteps the boundaries be- tween these domains. This is the case of NW and SW Iberia (Fig. 1), the Armorican Massif and the diferent domains that make up the Bohemian Massif, and implies that crustal units separated by hundreds or thousands of $\mathrm{km}$ in the Neopro- terozoic had been amalgamated before Middle 


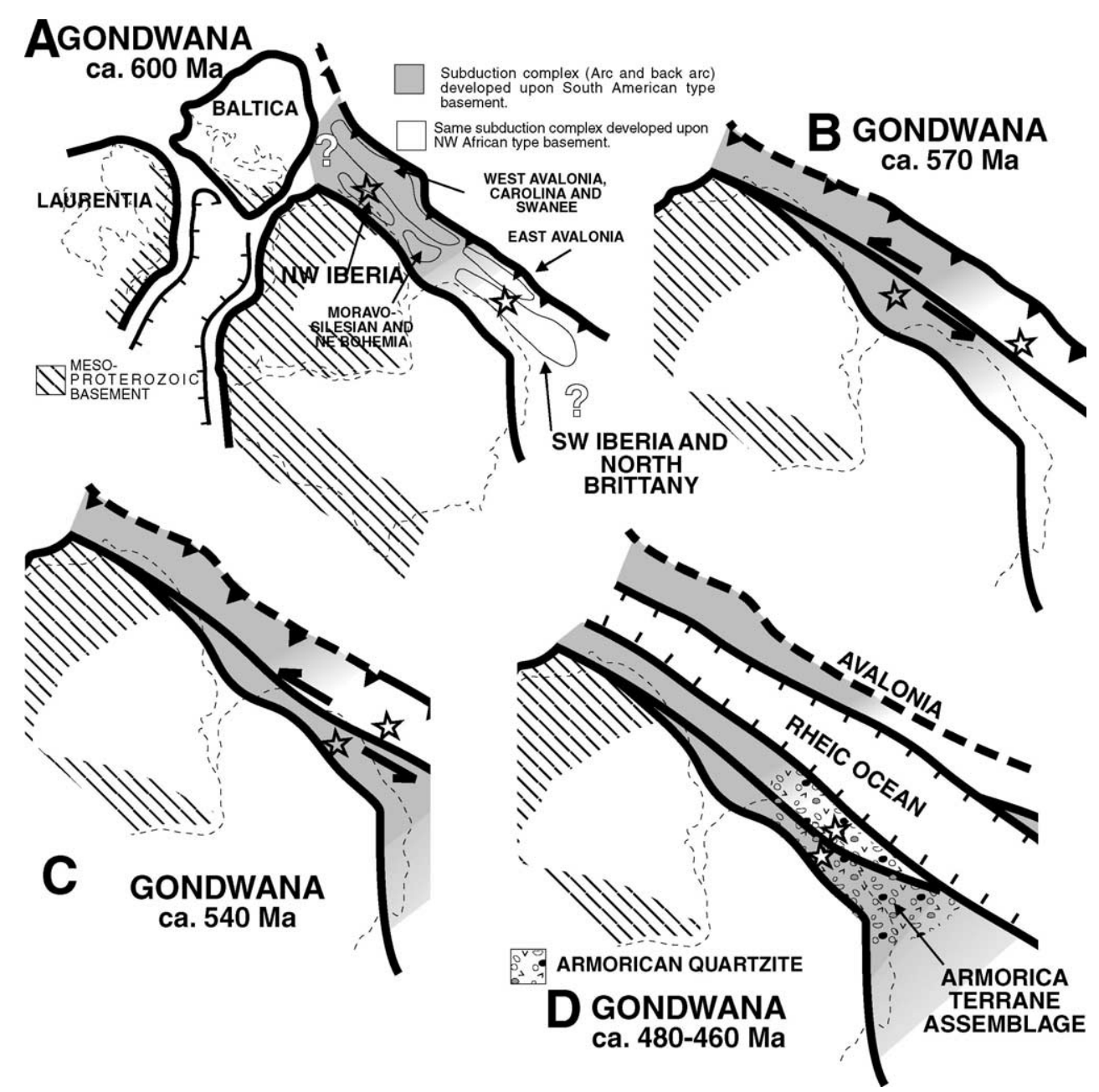

Fig. 4. Proposed Neoproterozoic terrane distribution (A,B) and early Palaeozoic tectonic evolution (C,D) along the northern Gondwanan margin (see text for details). Stars represent the hypothetical location of NW Iberia and SW Iberia/Brittany. Fig. 4A modifiedafter[1] and[6].

Ordovician times. This amalgamation is sup- ported by faunal, palaeomagnetic and geologic evidence which all indicate that NW and SW Iberia, the Armorican Massif and Bohemia had all been assembled adjacent to west Africa to form the Armorican realm (or Armorican Terrane Assemblage) by the Early to Middle Ordovician ([14] andreferencestherein).

If the Armorican quartzite was deposited upon an extensive peri-African continental platform [48,49], then the abundance of Mesoproterozoic zircons in this formation in NW Iberia [17] (Fig.

3) suggests that the source for these zircons had been tectonically transferred from an Amazonian realm beforethedeposition of thequartzite.

Therefore we propose that, prior to the Ordo- vician, NW Iberia (and other peri-Amazonian terranes of the Armorican Terrane Assemblage) were translated along the Gondwanan margin from an Amazonian realm towards the margin of west Africa. Later erosion of these tectonically trans- ported crustal fragments produced detritus containing Mesoproterozoic zircons that was incorporated into the Armorican quartzite.

Further, we suggest that this dispersal took place along major transform faults roughly paral- 
lel to the Gondwanan margin (Fig. 4) and pro- ceeded until the peri-Amazonian terranes had docked against those that had occupied a peri- west African location since the Neoproterozoic (Fig. 4C). This would explain why peri-Gond- wanan terranes with difering basement signatures are presently juxtaposed in the western European Variscan Belt.

This tectonic amalgamation gave birth to the Armorica Terrane Assemblage, which evolved as a single unit until its involvement in the Variscan- Appalachian collision in the late Palaeozoic. Geo- logical evidence for this docking may be recorded at the tectonic contact between the NW and the SW Iberian terranes [16,50] (Fig. 1), which we interpret as a reactivated early Palaeozoic terrane boundary. Deposition of the Armorican quartzite at ca $465 \mathrm{Ma}$ [51] provides a minimum age constraint for the docking between west Africa and Amazonia-derived terranes.

Shortly following (and/or partially overlapping) this dispersal of terranes along the Gondwanan margin a change in tectonic regime gave rise to extension on the margin and the birth of the Rheic Ocean. Rifting occurred between Armorica (an inboard terrane assemblage) and the more outboard Avalonia (Fig. 4D), which progressively separated from Gondwana until its docking to Laurentia-Baltica in the Silurian.

\subsection{Further remarks}

Margin-parallel strike-slip motion of terranes, as proposed here for north Gondwana, is observed in modern settings like the Cordilleran margin of North America [2] but has seldom been documented at this scale in ancient orogenic belts. We speculate that the periGondwanan terranes with Amazonian affinities moved towards the west African margin in a manner akin to that in which California (west of the St. Andreas fault) is moving towards Alaska. The west African margin, in which subduction may still have been active when the strike-slip motion was initiated on the Amazonian side [11], could have acted as a back-stop to Africa-wards drifting terranes. Thus the Armorica Terrane Assemblage could be regarded as a bundling of terranes generated by strike-slip motion along the northern Gondwanan margin.

\section{Conclusions}

Detrital zircon ages in Neoproterozoic and early Palaeozoic sedimentary rocks from western Europe provide a record of the geography and geodynamic evolution along the northern Gond- wanan margin from ca 600 to 480 Ma. Such studies can provide first-order constraints on the histories of terrane dispersal and accretion, particularly in those cases where the geological record has been obscured by major orogenic events or subsequent plate reorganisation.

Constraining these processes in ancient orogenic belts can significantly improve our understanding of the geological record. We have demonstrated that $\mathrm{U}-\mathrm{Pb}$ geochronology of detrital zircon populations can be used to test the role of transform margins in ancient orogens and crus- tal growth episodes.

\section{Acknowledgements}

This study was funded through an EU SYSResource (TMR Program) grant to J.F.S. J.F.S. also acknowledges a 'Contrato de Reincorporación para Doctores y Tecnólogos' from the Spanish Ministry of Education. Additional funding to

G.G.A. was provided by Project BTE2000-1490- C02-01 of the Spanish Ministry of Education. The Electron Microscopy and Mineral Analysis Division, Frances Wall, and Lyanne Houseago (NHM) are kindly acknowledged for technical and logistic support. A. Azor and I. Expósito are thanked for useful sampling advise. Brendan Murphy, Sue Keay and an anonymous reviewer are kindly acknowledged for very insightful and constructive reviews. This paper is a contribution to IGCP Project 453.[BARD] 


\section{References}

[1] R.D. Nance, J.B. Murphy, Contrasting basement signtures and the palinspastic restoration of peripheral oro- gens: an example from the Neoproterozoic Avalonian Cadomian Belt, Geology 22 (1994) 617-620.

[2] P.J. Pacthett, C.G. Chase, Role of transform continental margins in major crustal growth episodes, Geology 30 (2002) 39-42.

[3] G.E. Gehrels, G.M. Ross, Detrital zircon geochronology of Neoproterozoic to Permian miogeoclinal strata in Brit- ish Columbia and Alberta, Can. J. Earth Sci. 35 (1998) 1380-1401.

[4] T.R. Ireland, T. Fiattman, C.M. Fanning, G.M. Gibson, W.V. Preiss, Development of the early Paleozoic Pacific margin of Gondwana from detrital-zircon ages across the Delamerian orogen, Geology 26 (1998) 243-246.

[5] J.D. Keppie, D.W. Davies, T.E. Krogh, U-Pb geochrono- logical constraints on Precambrian stratified units in the Avalon Composite Terrane of Nova Scotia, Canada: tectonic implications, Can. J. Earth Sci. 35(1998) 222- 236.

[6] J. Fernández-Suárez, G. GutierrezAlonso, G.A. Jenner, M.N. Tubrett, New ideas on the Proterozoic-Early Paleo- zoic evolution of NW Iberia. Insights from U-Pb detrital zircon ages, Precambrian Res. 102 (2000) 185-206.

[7] K. Yamasita, R.A. Creaser, M.E. Villeneuve, Integrated $\mathrm{Nd}$ isotopic and U-Pb detrital zircon systematics of clastic sedimentary rocks from the Slave Province, Canada: evi- dence for extensive crustal reworking in the early- to mid- Archean, Earth Planet. Sci. Lett. 174 (2000) 283-299.

[8] J.W.F. Ketchum, S.E. Jackson, N.G. Culshaw, S.M. Barr, Depositional and tectonic setting of Paleoproterozoic Lower Aillik group, Makkovik Province, Canada: evolu- tion of a passive margin-foredeep sequence based on pet- rochemistry and $\mathrm{U}-\mathrm{Pb}$ (TIMS and LAM-ICP-MS) geo- chronology, Precambrian Res. 105(2001)331-356.

[9] R.F. Berry, G.A. Jenner, S. Mefre, M.N. Tubrett, A North American provenance for Neoproterozoic to Cam- brian sandstones in Tasmania?, Earth Planet. Sci. Lett. 192 (2001) 207-222.

[10] J.B. Murphy, R.D. Nance, Sm-Nd isotope systematics as tectonic tracers: an example from West Avalonia in the Canadian Appalachians, Earth Sci. Rev. 1246(2002) in press.

[11] J.B. Murphy, R.A. Strachan, R.D. Nance, K.D. Parker, M.B. Fowler, Proto-Avalonia: A 1.2-1.0 Ga tectonother- mal event and constraints for the evolution of Rodinia, Geology 28 (2000) 1071-1074.

[12] H. Kemmitz, R.L. Romer, O. Oncken, Gondwana break- up and the northern margin of the Saxothuringian belt (Variscides of Central Europe), Int. J. Earth Sci. 91 (2002) 246-259.

[13] I.W.D. Dalziel, Neoproterozoic-Paleozoic geography and tectonics: Review, hypothesis, environmental speculation, Geol. Soc. Am. Bull. 109(1997)16-42.

[14] J.A. Tait, V. Bachtadse, W. Franke, H.C. Sofel, Geodya- mic evolution of the European Variscan fold belt: paleo- magnetic and geological constraints, Geol. Rundschau 86 (1997) 585-598.
[15] C.McA. Powell, S.A. Pisarevsky, Late Neoproterozoic assembly of East Gondwana, Geology30(2002)3-6.

[16] L. Eguiluz, J.I. Gil Ibarguchi, B. Abalos, A. Apraiz, Superposed Hercynian and Cadomian orogenic cycles in the Ossa Morena Zone and related areas of the Iberian Massif, Geol. Soc. Am. Bull. 112 (2000) 1398-1413.

[17] J. Fernández-Suárez, G. Gutiérrez Alonso, R. Cox, G.A. Jenner, Assembly of the Armorica microplate: A strike- slip terrane delivery? Evidence from U-Pb ages of detrital zircons, J. Geol. 110 (2002) 619-626.

[18] E.A. Nagy, S.D. Samson, R.S. D’Lemos, U-Pb geochro- nological constraints on the timing of Brioverian sedimen- tation and regional deformation in the St. Brieuc region of the Neoproterozoic Cadomian orogen, northern France, Precambrian Res. 116 (2002) $1-17$.

[19] T.E. Jefries, S.E. Jackson, H.P. Longerich, Application of a frequency quintupled Nd:YAG source (Lamb- da = $213 \mathrm{~nm}$ ) for laser ablation inductively coupled plas- ma mass spectrometric analysis of minerals, J. Anal. At. Spectrosc. 13 (1998) 935-940.

[20] T.E. Jefries, J. Fernández-Suárez, Zircon dating 3213nm versus 266nm Nd:YAG lasers, Application Note, New Wave ResearchMerchantek Products, 2001, 2 pp.

[21] M. Wiedenbeck, P. Alle, F. Corfu, W.L. Grin, M. Mei- er, F. Orbeli, A. Von Quadt, J.C. Roddick, W. Spiegel, Three natural zircon standards for U-Th-Pb, Lu-Hf, trace element and REE analyses, Geostandards Newslett. 19 (1995) 1-23.

[22] T. Andersen, Correction for common-lead in U-Pb anal- yses that do not report ${ }^{204} \mathrm{~Pb}$, Chem. Geol. (2002) in press.

[23] W. Compston, I.S. Williams, C. Meyer, U-Pb geochronol- ogy of zircons from Lunar Breccia 73217 using a sensitive high-mass resolution ion microprobe, J. Geophys. Res. 89 (1984) B525-B534.

[24] W. Compston, Geological age by instrumental analysis, Mineral. Mag. 63 (1999) 297-311.

[25] B. Ordonez Casado, Geochronological studies of the Pre- Mesozoic basement of the Iberian Massif: The Ossa Mo- rena Zone and the allochthonous complexes within the Central Iberian Zone, ETH diss.12.940(1998)235pp.

[26] I. Horn, R.L. Rudnick, W.F. McDonough, Precise ele- mental and isotope ratio determination by simultaneous solution nebulization and laser ablation ICP-MS: appli- cation to U-Pb geochronology, Chem. Geol. 167 (2000) 405-425.

[27] K.R. Ludwig, On the treatment of concordant uranium- lead ages, Geochim. Cosmochim. Acta 62 (1998)665-676.

[28] B.V. Miller, S.D. Samson, R.S. D’Lemos, U-Pb geochro- nological constraints on the timing of plutonism, volca- nism, and sedimentation, Jersey, Channel Islands, UK, J. Geol. Soc. London 158 (2001) 243-252.

[29] R.D. Nance, M.D. Thompson (Eds.), Avalonian and re- lated PeriGondwanan Terranes of the circum-North At- lantic, Geol. Soc. Am. Spec.Publ.304, 1996.

[30] K.P. Hefernan, H. Admou, A.K. Jefrey, A. Saquaque, Anti-Atlas (Morocco) role in Neoproterozoic west Gond- wana reconstruction, Precambrian Res. 103(2000)89-96. 
[31] F.F. Alkmim, S. Marshak, M.A. Fonseca, Assembling west Gondwana in the Neoproterozoic: Clues from the Sao Francisco craton region, Brazil, Geology 29 (2001) 319-322.

[32] G. Rocci, G. Bronner, M. Deschamps, Crystalline base- ment of the west African craton, in: R.D. Dallmeyer, J.P. Lecorche (Eds.), The West African Orogens and Circum- Atlantic Correlatives, Springer, Berlin, 1991, pp. 31-61.

[33] W. Teixeira, C.C.G. Tassinari, U.G. Cordani, K. Kawa- shita, A review of the geochronology of the Amazonian craton, tectonic implications, Precambrian Res. 42 (1989) 213-227.

[34] Z.S. de Souza, A. Potrel, J.M. Lafon, F.J. Althof, M.M. Pimentel, R. Dall'Agnol, C.G. de Oliveira, $\mathrm{Nd}, \mathrm{Pb}$ and $\mathrm{Sr}$ isotopes in the Identidade Belt, an Archean greenstone belt of the Rio Maria region (Carajas srovince, Brazil): implications for the Archaean geodynamic evolution of the Amazonian craton, Precambrian Res. 109 (2001) 293-315.

[35] J.Y. Calvez, P.H. Vidal, Two billion year old relicts in the Hercynian beltof westernEurope,Contrib. Mineral.Pet- rol.65(1978)395-399.

[36] S.D. Samson, R.S. D’Lemos, U-Pb geochronology and Sm-Nd isotopic composition of Proterozoic gneisses, Channel Islands, UK, J. Geol. Soc. London 155 (1998) 609-618.

[37] A.N. Kouamelan, C. Delor, J.J. Peucat, Geochronological evidence for reworking of Archean terranes during the early Proterozoic (2.1 Ga) in the western Corte d'Ivoire (Man Rise-West African craton, Precambrian Res. 86 (1997) 177-199.

[38] A. Potrel, J.J. Peucat, C.M. Fanning, Archean crustal evolution of the West African craton; example of the Amsaga area (Reguibat Rise); U-Pb and Sm-Nd evidence for crustal growth and recycling, Precambrian Res. 90 (1998) 107-117.

[39] D. Thieblemont, C. Delor, A. Cocherie, J.M. Lafon, J.C. Goujou, A. Balde, M. Bah, H. Sane, C.M. Fanning, A 3.5 Ga granite gneiss basement in Guinea, further evidence for early Archean accretion within the west African cra- ton, Precambrian Res. 108 (2001) 179194.

[40] C.C.G. Tassinari, M.J.B. Macambira, Geochronological provinces in the Amazonian craton, Episodes 22 (1999) 174-182.

[41] E. Hegner, A. Krø ner, Review of Nd isotopic data and xenocrystic and detrital zircon ages from the pre-Variscan basement in the eastern Bohemian Massif: speculations on palinspastic reconstructions, in: W. Franke, V. Haak, O. Oncken, D. Tanner (Eds.), Orogenic Processes: Quantification and Modelling in the Variscan Belt, Geol. Soc. Spec. Publ. 179, 2000,pp. 167-175.

[42] G. Friedl, F. Finger, N.J. McNaughton, I.R. Fietcher, Deducing the ancestry of terranes: SHRIMP evidence for South Americaderived Gondwana fragments in cen- tral Europe, Geology 28 (2000) 1035-1038.

[43] D. Gebauer, I.S. Williams, W. Compston, The develop- ment of the central European continental crust since the early Archean based on conventional and ion-microprobe dating of up to 3.84 b.y. old detrital zircons, Tectonophy- sics 157 (1989) 81-96.

[44] M. Tichomirowa, H.J.Berger, E.A. Koch, B.V.Belyatski,

J. G $₫$ tze, U. Kempe, L. Nasdala, U. Schaltegger, Zircon ages of high-grade gneisses in the Eastern Erzgebirge (Central European Variscides) - constraints on origin of the rocks and Precambrian to Ordovician magmatic events in the Variscan foldbelt, Lithos 56 (2001) 303- 332.

[45] J.D. Keppie, J. Dostal, F. Ortega-Gutierrez, R. Lopez, A Grenvillian arc on the margin of Amazonia: evidence from the southern Oaxacan Complex, southern Mexico, Precambrian Res.112 (2001)165-181.

[46] T.F. Nagler, H.J. Schafer, D. Gebauer, Evolution of the Western European continental crust: implications from $\mathrm{Nd}$ and $\mathrm{Pb}$ isotopes in Iberian sediments, Chem. Geol. 121 (1995) 345-347.

[47] E. Mullane, The geochemistry of the South Portuguese Zone, Spain and Portugal, unpublished Ph.D. Thesis, University Southampton, UK, 1998, 260 pp.

[48] F. Paris, M. Robardet, Early Palaeozoic palaeobiogeog- raphy of the Variscan regions, Tectonophysics 177 (1990) 193-213.

[49] W.S. McKerrow, C.R. Scotese (Eds.), Palaeozoic Palaeogeography and Biogeography, The Geological Society Memoir 12, 1990, 435 pp.

[50] J.F. Simancas, D. Martmez Poyatos, I. Expø sito, A. Azor, F. Gonza lez Lodeiro, The structure of a major suture zone in the SW Iberian Massif: the Ossa More- na/Central Iberian contact, Tectonophysics 332 (2001) 295-308.

[51] J.L. Bonjour, J.J. Peucat, J.J. Chauvel, F. Paris, J. Cor- nichet, U-Pb zircon dating of the Early Paleozoic (Areni- gian) transgression in western Brittany (France): a new constraint for the Lower Paleozoic time-scale, Chem. Geol.72(1988)329-336. 